\title{
Design and Simulation of a Ridge Horn Antenna
}

\author{
Lei Xie, Wei He*, Wenpe Li \\ School of Physics and Electronic Information, Yunnan Normal University, Kunming, China \\ *Corresponding author: he99wei@aliyun.com
}

Keywords: dual-ridge, horn antenna, Structural characteristics, simulation

\begin{abstract}
The horn antenna has large bandwidth, superior directionality, easy design and processing, and high gain. It has become an ultra-wideband antenna that can be practically applied in many occasions. The ridged horn antenna has an ultra-wideband, wide-band, uniform pattern. Moreover, it has a higher gain and is widely used as a feed of a high gain antenna or an array element of an array antenna. This paper introduces the structural characteristics of the double-ridged horn antenna from many aspects, and designs a double-ridged horn antenna. And through electromagnetic simulation of HFSS, the results show that the double-ridged horn antenna has good directivity in the frequency range of $20 \mathrm{GHz} 26 \mathrm{GHz}$. The results showed that the gain reaches 18 $\mathrm{dB}$ at $24 \mathrm{GHz}$, and the main flap of the three-dimensional gain pattern is not cracked.
\end{abstract}

\section{Introduction}

Since the antenna, the device used to receive and radiate electromagnetic waves, has been designed by Hertz and Marconi. It has begun to play a key role in daily communication. Nowadays, it has become an indispensable device to ensure people's normal communication [1]. Ultra-wideband antenna technology is a hot spot in antenna research at home and abroad. The technology was mainly used in the military field in the early days, and it has outstanding contributions in radar monitoring, anti-stealth technology, and electronic countermeasures. In recent years, UWB technology has gradually been introduced into the civilian field, and has been widely used in the fields of broadband communication, spread spectrum communication, ground penetrating radar, field measurement, and electromagnetic compatibility [2].

At present, the most common ultra-wideband antennas can be roughly divided into small-element antennas such as double-cone and disc-cone antennas and bow-tie antennas according to their shapes; frequency-independent antennas such as helical antennas and log-periodic antennas; and ridged horn antennas and Vivaldi antennas. The evolution of the horn antenna and so on. Not only the operating frequency of an ordinary horn antenna is affected by the size of the transmission waveguide, but also limited by the gain requirement for the speaker. In order to increase the bandwidth of the horn antenna and reduce the main mode cutoff frequency and its impedance characteristics, the ridge is usually used to improve the transmission characteristics of the horn antenna [3]. This paper designs a double-ridged horn antenna. And electromagnetic simulation of it by HFSS (High Frequency Structure Simulation). The dual-ridged horn antenna has excellent radiation characteristics in the operating frequency range of $20 \mathrm{GHz}$ to $26 \mathrm{GHz}$.

\section{Double Ridge Horn Antenna Structure}

The ordinary horn antenna can be regarded as extending outward from the waveguide. From the broadband characteristics of the ridge waveguide, the ridge structure is added to the waveguide segment and the horn segment of the horn antenna. Thereby it can obtain a double-ridge horn antenna with a widened band. The structure of the double-ridged horn antenna is shown in Figure 1. The structure can be divided into a coaxial feed joint, a waveguide section, a horn section, and a double ridge [4]. 


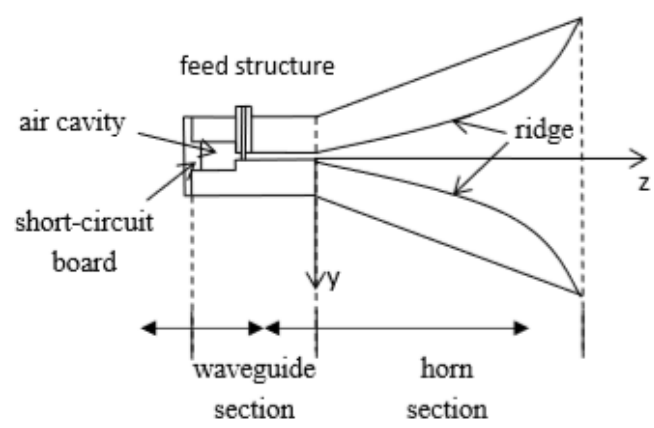

Fig.1. Cross-section of double-ridged horn antenna

The structure of the waveguide section is complicated. And the straight waveguide section is from the short-circuit board to the feed. Its function is to filter out the TE20 mode excited in the waveguide. The length of this segment has a direct influence on the performance of the whole antenna. The distance from the feed probe to the short-circuit version is less than half of the working wavelength. From the feeding section to the neck of the horn, it is a ridge waveguide section. The ridged waveguide is mainly used to reduce the cutoff frequency of the main mode transmission to achieve wideband characteristics. The cross-sectional dimension of the ridge waveguide portion is gradually increased from the feeding portion to the neck of the horn, and the ridge spacing is also linearly increased [5]. This section is approximated as a ridged horn.

There is an air cavity between the ridge waveguide and the short circuit plate. The function of the reflection cavity is to suppress the $T E_{20}$ electromagnetic wave of the mode in the waveguide. This can make the main mode (TE10 mode single mode transmission has a larger bandwidth) and play the role of widening the frequency band [6].

Horn segment: in order to effectively suppress the transmission of higher-order modes generated during impedance conversion, the length of the horn is at least half the wavelength of the lowest operating frequency. The calibre size of the horn is determined by the phase difference between the gain and the aperture surface. Good matching effect when the horn segment impedance is as follows.

$$
\begin{cases}Z=Z_{\infty} e^{k z} & 0 \leq Z \leq L / 2 \\ Z=377+Z_{\infty}\left(1+e^{k(L-z)}\right) & L / 2 \leq Z \leq L\end{cases}
$$

$$
Z_{L / 2}=\frac{Z_{0}+Z_{L}}{2}
$$

Where $L$ the length of the horn segment, and $k$ is a constant (it can be determined by equation (2)).

The shape curve of the ridge structure is generally changing by as follow:

$$
y(z)=A e^{k z}+C z
$$

\section{Design Principle and Method}

First to define the size of the waveguide, and the cross-sectional view is shown in Figure 2.

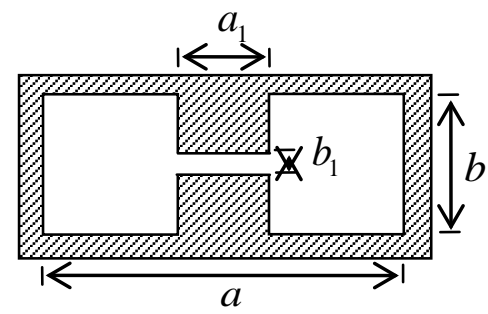

Fig.2. Ridge waveguide structure 
A schematic cross section of the ridge waveguide portion is shown in Figure 2. The cross-section dimension of the waveguide is $a \times b$, where the ridge width is $a_{1}$, and the ridge spacing is $b_{1}$. The design is mainly based on the ridge waveguide theory. The function of the ridge waveguide is to transmit the electromagnetic wave fed by the coaxial line, so that when entering the ridge horn, a stable electromagnetic wave can be formed. furthermore, its size is also related to the cutoff frequency of the antenna working.

According to the antenna frequency band requirements of $20 \mathrm{GHz}$ to $26 \mathrm{GHz}$, the main mode cutoff wavelength is $\lambda^{\prime}=3.20 \mathrm{~cm}$, and $T E_{20}$ mode cutoff wavelength is $\lambda^{\prime \prime}=1.11 \mathrm{~cm}$. From this, we can get the size of the waveguide. Finally, we chose the length of the wide side of the waveguide is $a=1.2 \mathrm{~cm}$, and the narrow side length is $b=0.556 \mathrm{~cm}$, and the ridge width is $a_{1}=0.3 \mathrm{~cm}$, and the ridge spacing is $b_{1}=0.236 \mathrm{~cm}$.

The excitation of the horn feed structure are used $50 \Omega$ feeder and $N$-type connector. We pass the coaxial feed through the center of the first ridge, and the center conductor feeds the second ridge across the gap of the ridge. Thereby a monopole radiator is formed. In the horn waveguide section, the ridge waveguide between the feed and the neck of the horn has a linear increase in cross-sectional area to avoid impedance mismatch due to structural abrupt changes [4]. Therefore, we use the italic structure for the ridge waveguide wall. The size of both sides of the ridge waveguide is linearly increased. The cross-sectional view is shown in Figure 3.

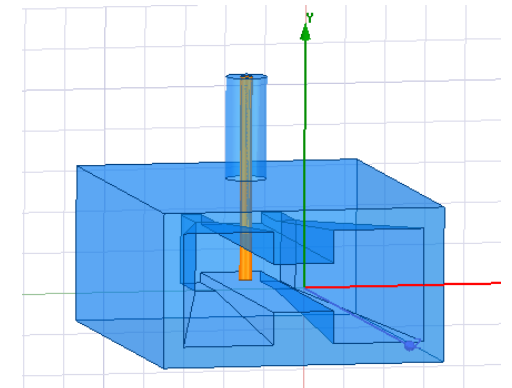

Fig.3. Feed ridge waveguide section

The shape of the ridge is designed according to the impedance matching principle of the transmission line, so that the impedance inside the horn can smoothly transition. The horn Is a standard gain antenna. The size of the horn can be calculated from the optimal pyramid horn design equation [7] by given gain, operating wavelength and waveguide size:

$$
A^{4}-a A^{3}+\frac{3 b G \lambda^{2}}{8 \pi \varepsilon_{a p}} A=\frac{3 G^{2} \lambda^{2}}{32 \pi^{2} \varepsilon_{a p}^{2}}
$$

For the best gain pyramid horn, we usually use 50\% of the aperture as efficiency value:

$$
G=0.51 \frac{4 \pi}{\lambda^{2}} A B
$$

The relationship between the diameter of the horn and the metal plate length radiating section is as follows.

$$
\begin{aligned}
& A=\sqrt{3 \lambda R_{1}} \\
& B=\sqrt{2 \lambda R_{2}}
\end{aligned}
$$

Bring the parameter of $\varepsilon_{a p}=0.51$ into formula (4) to find $A$. Bring the obtained value of $A$ into the formula (5) to find $B$. Finally, we can calculate the value of each parameters of the horn: $A=5.608 \mathrm{~cm}, B=4.347 \mathrm{~cm}, R_{1}=8.387 \mathrm{~cm}, R_{2}=7.559 \mathrm{~cm}$. Shape curve formula combined with ridge structure (3): $y(z)=A e^{k z}+C z, A=b_{1} / 2, C=0.015$. The ridge structure curve can be obtained 
after the data is brought in.

$$
y(z)=0.00118 e^{43.490 z}+0.015 z
$$

\section{Simulation of Double Ridge Horn Antenna}

According to the design requirements of this paper, the double-ridged horn antenna shown in Figure 4. It was designed by HFSS. The width of the ridge waveguide is $a=1.2 \mathrm{~cm}$. The length of the narrow side is $b=0.556 \mathrm{~cm}$. Ridge width is $a_{1}=0.3 \mathrm{~cm}$. Ridge spacing is $b_{1}=0.236 \mathrm{~cm}$. The length of the horn is $5.608 \mathrm{~cm}$. The width is $4.347 \mathrm{~cm}$, and the length is $6.592 \mathrm{~cm}$. The length of the waveguide is $1.563 \mathrm{~cm}$.

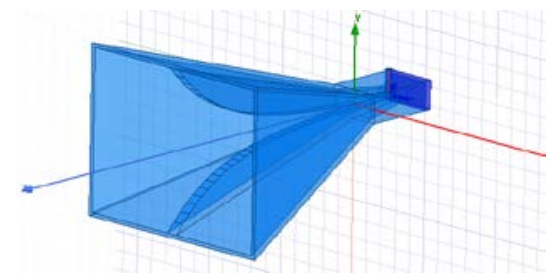

Fig.4. Double ridge horn antenna module

The gain of the E-plane and the H-plane of the dual-ridge horn antenna in $24 \mathrm{GHz}$ is as shown in Figure 5. The gain directions of the E-plane and H-plane in the polar coordinate system are shown in Figure 6. The three-dimensional gain pattern is shown in Figure 7.

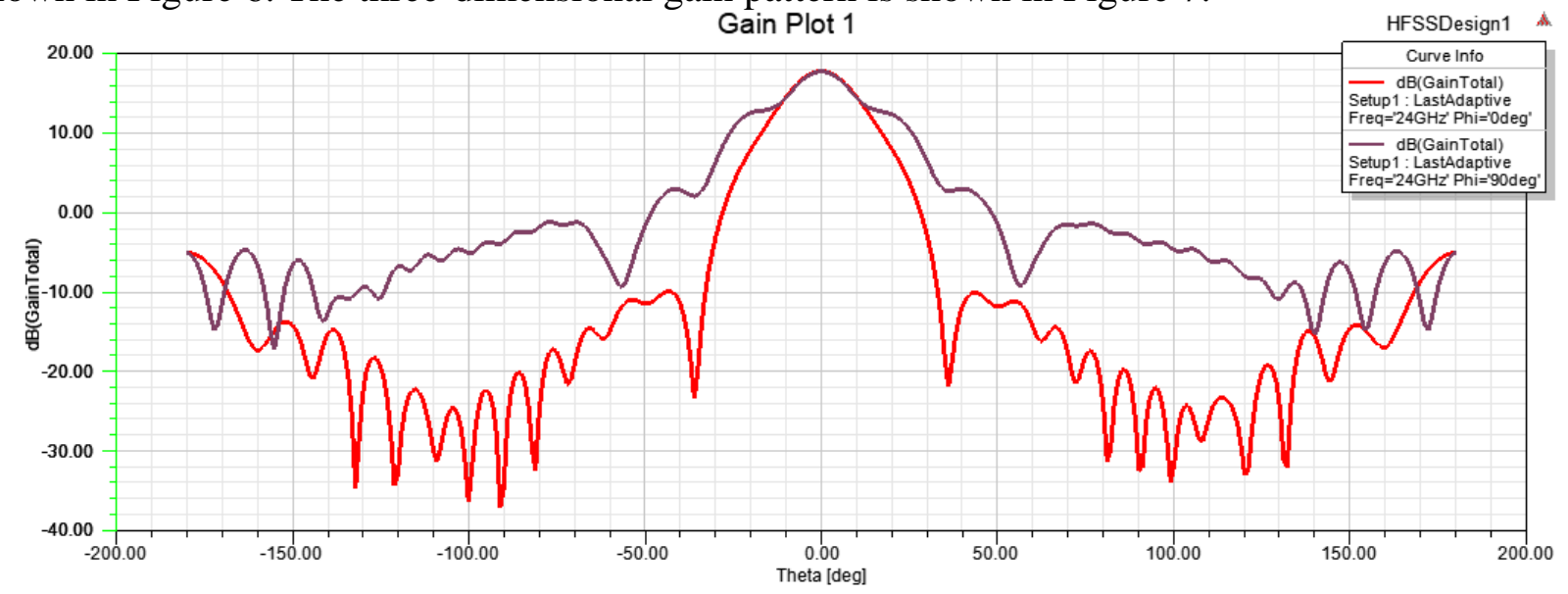

Fig.5. E-plane and H-plane gain pattern
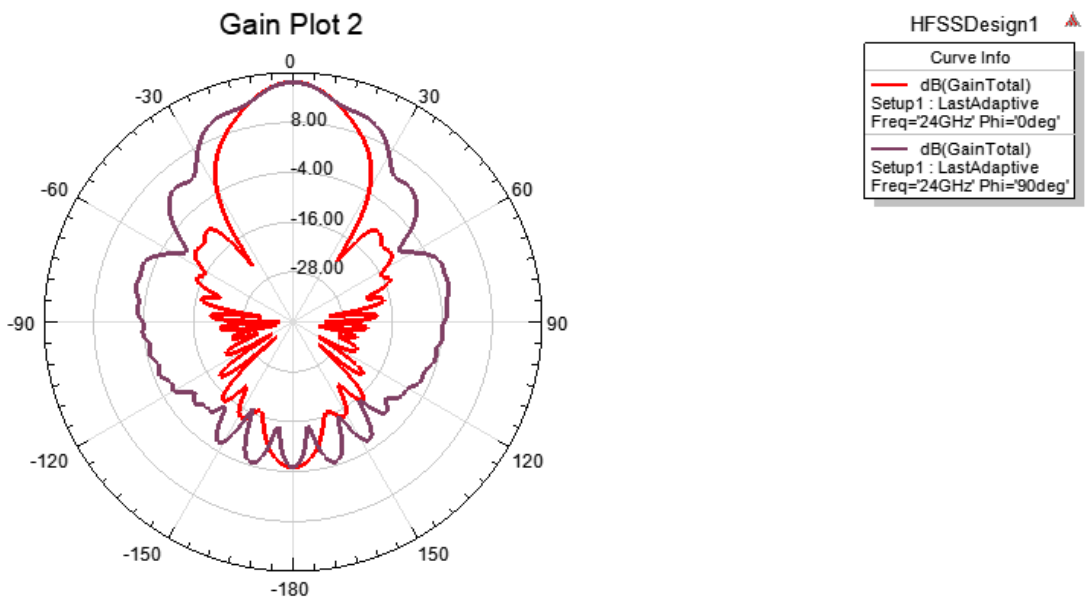

Fig.6. Gain pattern of E-plane and H-plane in polar coordinate system 

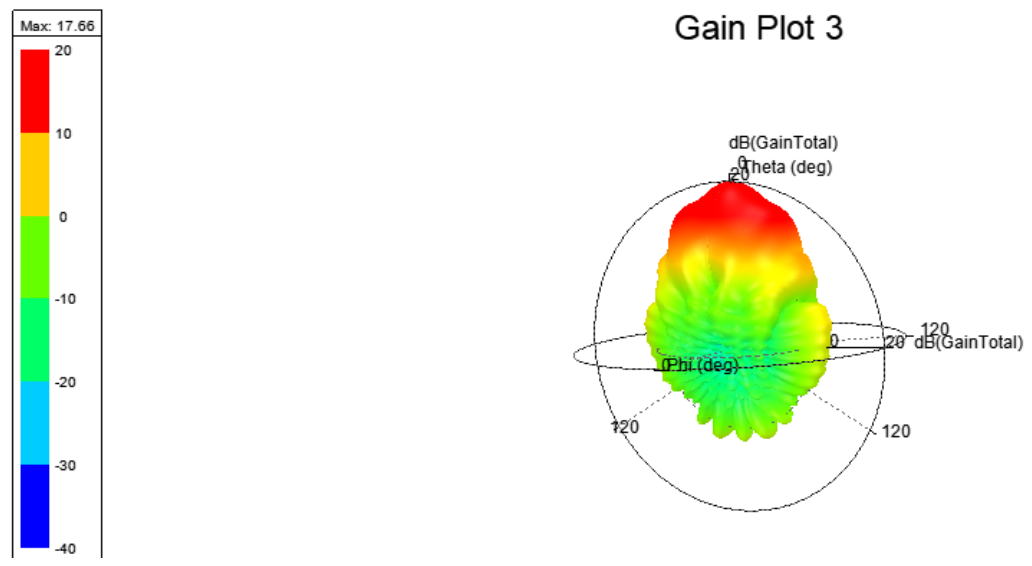

Fig.7. Three-dimensional gain pattern

\section{Summary}

This article starts from the structure of the double-ridged horn antenna and the working principle of each part. According to actual needs, a broadband double-ridged horn antenna was designed. And wrote the design steps in detail. Draw the designed double-ridged horn antenna in simulation software HFSS (High Frequency Structure Simulation) according to the actual parameters. And simulated simulation experiments. The result shows that the antenna has a gain of $18 \mathrm{~dB}$ at $24 \mathrm{GHz}$ operating frequency. It has superior gain directivity. Overall simulation and measurement results are ideal, in line with actual needs. It has certain reference value for the design of such antennas in the future.

\section{Acknowledgements}

This project was supported by National Natural Science programs (51267021) and College students' innovative entrepreneurial training programs (2015).

\section{References}

[1] Yue Zhenzhen, Design of a broadband double-ridged horn antenna [D]. Xidian University, 2017.

[2] Tenigeer, Research on ultra-wideband dielectric-loaded horn antenna [D]. Harbin Institute of Technology, 2014.

[3] Liu li, Theory analysis and simulation of electrical properties for broadband dual-polarized quadruple-ridged horn antenna [J]. System Simulation Technology, 2017, (4): 48.

[4] Zhao Jia-fei, Wang Xue-tian, Wang Wei, Simulation and design of wide band double-ridged horn antenna [J]. Journal of Microwaves, 2014 (5) 264-266.

[5] Yang kang, Design Method of Ultra-wideband Double-ridged Horn Antenna[D]. China Ship Research and Development Academy.2015.

[6] Zhao jianbei. Research on Characteristics of Ultra Wideband TEM Ridged Horn Antenna [D]. Harbin Institute of Technology.2013.

[7] Warren L, Stutzman Gary A, Thiele. Antenna Theory and Design, M. Zhu Shouzheng, Translation. Beijing, People Post Press, 2006 (2) 291-292. 